\title{
Sarcopenia in cirrhosis: A practical overview
}

\author{
Authors: Amritpal Dhaliwal ${ }^{A}$ and Matthew ] Armstrong ${ }^{B}$
}

Patients with liver cirrhosis and, in particular, those with liver failure are at high risk of reduced muscle mass and strength/ function, otherwise known as sarcopenia. Sarcopenia is a complex, multifactorial (poor nutritional intake, protein catabolism, physical inactivity) chronic condition, which increases the risk of liver-related morbidity and mortality. Early recognition and tailored management incorporating high protein diets and combination aerobic/resistance exercise can ameliorate the complications associated with sarcopenia in cirrhosis. This review provides an overview of the epidemiology, pathogenesis, assessment tools and management of sarcopenia in cirrhosis.

\section{Introduction}

Sarcopenia is a muscle wasting syndrome that is defined by a reduction in muscle mass on cross-sectional imaging. ${ }^{1}$ The definition of sarcopenia is still under debate in cirrhosis, but for the purpose of this review it also includes a reduction in muscle strength and/or function. ${ }^{1}$ Cirrhosis is a catabolic state in which muscle protein breakdown exceeds synthesis resulting in sarcopenia. ${ }^{2}$ It is associated with higher rates of complications of cirrhosis (eg hepatic encephalopathy and infections), hospital admissions and premature mortality. In addition, it is associated with poorer clinical outcomes after liver transplantation (eg rejection, length of hospital stay and mortality), in addition to reduced quality of life and lack of functional independence., ${ }^{3,4}$ As liver transplantation remains the only cure for patients with decompensated cirrhosis, early recognition and management of sarcopenia is a critical aspect of the care of patients with cirrhosis.

\section{Epidemiology}

The prevalence of sarcopenia in cirrhosis ranges from $30-70 \%$, depending on the diagnostic tools utilised and the severity of the underlying liver disease. ${ }^{5}$ Increasing rates of computed tomography (CT)-defined sarcopenia are observed with worsening liver disease

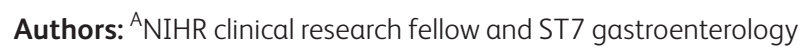
and hepatology registrar, Queen Elizabeth Hospital Birmingham, Birmingham, UK, National Institute for Health Research Birmingham Biomedical Research Centre, Birmingham, UK and University of Birmingham, Birmingham, UK; ${ }^{\mathrm{B}}$ consultant transplant hepatologist, Queen Elizabeth Hospital Birmingham, Birmingham, UK and National Institute for Health Research Birmingham Biomedical Research Centre, Birmingham, UK severity, as defined by the Child-Pugh score $(A=10 \%$; $B=34 \%$; $\mathrm{C}=54 \%)^{6}$ There are many different aetiologies of cirrhosis in the UK. The most common cause has consistently been alcohol-related liver disease (ARLD) over the last decade, with a sharp increase in non-alcoholic fatty liver disease (NAFLD) due to the epidemic of obesity and diabetes. ' Liver disease aetiology is important in sarcopenia, as there are liver disease-specific drivers that may potentiate the progression of sarcopenia; for example, alcohol promotes skeletal muscle protein breakdown, cholestasis promotes fat and vitamin D malabsorption in primary biliary diseases, whereas sarcopenia and NAFLD share similar pathological mediators, such as chronic inflammation, insulin resistance and physical inactivity. $2,8,9$ Challenges arise when the prevalence of sarcopenia may be masked by the coexistence of morbid obesity (termed 'sarcopenic obesity'), which is most notable in patients with NAFLD.

\section{Key points}

Sarcopenia is prevalent and is defined as a reduction in muscle mass ('muscle wasting state') and is often accompanied by a reduction in muscle strength and function. It increases the risk of morbidity (liver failure and hospitalisation) and mortality.

Routine assessment of sarcopenia is highly recommended and involves measures of muscle mass (computed tomography at the L3 vertebra level; mid-arm muscle circumference) $+/$ - muscle strength and function (hand grip strength; chair stands).

Nutritional management includes high protein intake (1.2-2.0 g/kg/day), 2-3 hourly meals and late-evening snacks ( $50 \mathrm{~g}$ carbohydrate) in order to protect against protein catabolism and accelerated starving.

A combination of body-weight resistance and aerobic exercise are recommended to improve sarcopenia and exercise capacity.

Pharmacotherapy (eg vitamin D; testosterone in males) and experimental agents (eg myostatin inhibitors) are promising therapeutic options in the future, but require further study.

KEYWORDS: Sarcopenia, cirrhosis, liver failure, muscle, nutrition

DOI: $10.7861 /$ clinmed.2020-0089 


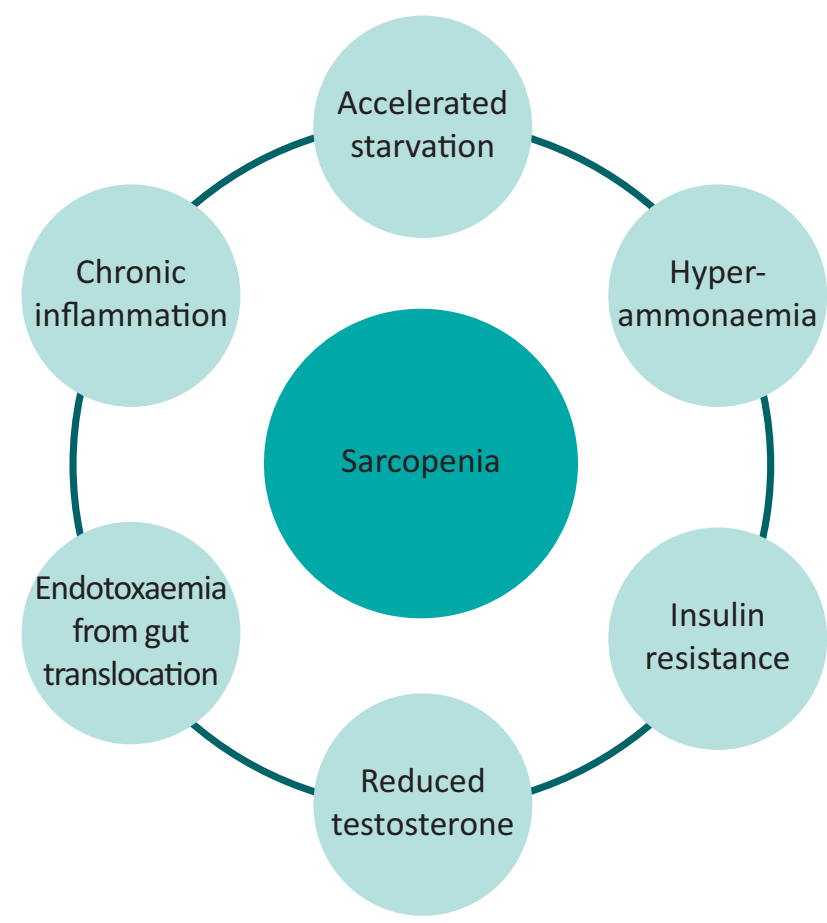

Fig 1. Main drivers of sarcopenia in liver cirrhosis.

\section{Pathogenesis}

The mechanisms driving sarcopenia in cirrhosis are complex and multifactorial (Fig 1). The imbalance between protein catabolism and synthesis occurs due to reduced intake, increased breakdown and increased energy expenditure. ${ }^{10,11}$ Reduced oral intake occurs due to reduced appetite, early satiety, delayed gut motility, altered cognition secondary to hepatic encephalopathy, medication side effects (ie opioids), low mood/motivation and zinc deficiency (dysguesia). ${ }^{12}$ Patients with cirrhosis are in a state of 'accelerated starvation' that is an impaired adaptive response to fasting, whereby 10 hours of fasting approximates to 3 days of starvation in a healthy individual. $2,13,14$ This is because the body has to find alternative routes to provide glucose for energy, as cirrhosis results in chronic depletion of hepatic glycogen stores. Therefore, in prolonged fasting states (ie sleeping) substrates for gluconeogenesis are taken from excessive fatty acid breakdown (acetyl coenzyme A (COA)) and muscle protein breakdown (branched-chain amino acids (BCAA)), with resultant sarcopenia. $^{2,13}$

Hepatic encephalopathy, a consequence of impaired liver function and of shunting of blood around the liver, also plays a role in the development of sarcopenia. Not only does encephalopathy result in physical inactivity, but hyperammonaemia upregulates myostatin, which is a key inhibitor of protein synthesis and muscle regeneration. In addition, the muscle is a key extra-hepatic site for removal of excess ammonia, but as a result stores of BCAA (in the form of leucine) are depleted and are not available for protein synthesis. 2,13

Insulin also plays a key role in preventing protein (catabolism) and adipose (lipolysis) breakdown. In an insulin resistant state, commonly found in cirrhosis and especially NAFLD, the opposite occurs in muscle and adipose tissue, with reductions in protein synthesis, increases in protein catabolism and promotion of lipotoxicity, respectively. ${ }^{15}$ Therefore, forming the perfect environment to promote sarcopenia. Other hormone changes, such as low testosterone secondary to hypogonadism in males with cirrhosis, also potentiate sarcopenia via protein dysregulation. $^{2}$

\section{Assessment of sarcopenia}

There are several different modalities to assess each component of sarcopenia, summarised in Table 1, but the field lacks a single, safe and reproducible assessment tool (ie gold standard) to define sarcopenia.

\section{Muscle mass}

$>$ Computed tomography: This is the most used and validated modality to evaluate muscle mass, with particular focus in cirrhosis on the skeletal muscle index (SMI) at the level of the 3rd lumbar vertebra. It is the only modality that has validated sexspecific cut-offs, notably $<50 \mathrm{~cm}^{2} / \mathrm{m}^{2}$ in men and $<39 \mathrm{~cm}^{2} / \mathrm{m}^{2}$ in women. The challenges with $\mathrm{CT}$, which include cost and repeated radiation exposure, mean that its utility in sarcopenia will be limited to those patients having imaging as part of routine care (ie cancer screening or portal vein thrombosis).

$>$ Mid-arm muscle circumference (MAMC): MAMC (in cm) is obtained by measuring the mid-arm circumference (MAC; in $\mathrm{cm}$ ) and tricep skin fold (TSF; in mm). These measures are cheap, quick and can be safely used in routine clinical practice to assess repeated measures of sarcopenia. When used by appropriately trained individuals, it has good intra- and inter-observer reproducibility and has been shown to predict mortality. ${ }^{10}$

Table 1. Summary of tests used to assess sarcopenia (muscle mass, strength, function)

\section{Muscle mass}

Computed tomography (CT) L3 skeletal muscle index Magnetic resonance imaging (MRI)

Mid-arm muscle circumference (MAMC)

Dual-energy X-ray absorptiometry (DEXA)

Muscle ultrasound

Bioimpedance analysis (BIA) ${ }^{a}$

\section{Muscle function}

Short physical performance battery test (SPPB)

Gait speed

Timed get-up-and-go test ${ }^{a}$

Liver Frailty Index (LFI)

Aerobic exercise capacity: ${ }^{\text {b }}$

6-minute walk distance (6MWD)

Cardiopulmonary exercise testing (CPET)

\section{Muscle strength}

Handgrip strength (HGS)

Knee flexion/extension peak torque strength ${ }^{c}$ Liver Frailty Index (LFI)

\footnotetext{
${ }^{a}=$ less frequently used tests; ${ }^{b}=$ separate entity but relies on muscle function; ${ }^{c}=$ research-based tests that are not yet validated in clinical practice.
} 
> Ultrasound quadriceps: Evaluation of vastus lateralis has been gaining increasing favour in the elderly population as it is accessible, cost effective and without any radiation risk. With the appropriate training, it has high levels of intra- and inter-observer reliability, but validation is required in cirrhosis. In addition to muscle mass, ultrasound can measure muscle quality and, in particular, fat deposition (myosteatosis) to aid with understanding why some individuals have preserved muscle mass but reduced function. ${ }^{5,16}$

> Others: Dual-energy X-ray absorptiometry (DEXA) and bioimpedance are both measures of muscle mass, but are expensive, have radiation exposure and, in presence of fluid retention, are less accurate. ${ }^{1,11}$

\section{Muscle strength}

The most frequently used modality is hand grip strength (HGS) as it is simple and can be used for repeated measures. It is recommended by the recent European guidelines in the assessment of patients with cirrhosis. HGS has been shown to be a better predictor of adverse clinical outcomes than CT measures of muscle mass and model for end-stage liver disease (MELD), with improvements in HGS as small as $1 \mathrm{~kg}$ representing significant reductions in mortality. ${ }^{1,10,11}$

\section{Muscle function}

> Short physical performance battery (SPPB): SPPB consists of chair stands, gait speed and balance (each scored out of 4; with a maximal score of 12) and a score $<10$ increases the odds of mortality by 2.5 . One limitation is that the SPPB has a ceiling effect, in that no improvements can be observed beyond 12/12.

> Liver Frailty Index (LFI): LFI is a liver disease-specific, continuous variable (ie no ceiling or floor effect), which replaces gait speed with HGS and is sub-categorised into frail $(>4.4)$, pre-frail (3.24.4) and robust ( $<3.2)$. LFI is the best studied outpatient measure of muscle strength and function (the key components of frailty) to date in the setting of liver transplantation, and is an independent predictor of both pre- and post-transplant mortality. ${ }^{17}$
$>$ Aerobic exercise capacity: This relies on muscle function, and can be assessed using the easy-to-apply, objective 6-minute walk distance (6MWD) or the more expensive, time-consuming cardiopulmonary exercise test (CPET). The latter requires expensive equipment (treadmill, cycle ergometer) and specialist interpretation of the ventilatory anaerobic threshold (AT) and the peak exercise oxygen uptake (peak $\mathrm{VO}_{2}$ ). A 6MWD of less than 250 m represents poor muscle function/exercise capacity and is associated with two-fold increased mortality in patients on the transplant waiting list. Every $100 \mathrm{~m}$ decrease in the 6MWD represents an almost $50 \%$ increase in waitlist mortality, independent of liver disease severity. 17,18

\section{Intervention}

The management of sarcopenia involves a multi-dimensional approach (Fig 2); with specific focus on lifestyle, nutrition, exercise and adjunct pharmacotherapy.

\section{Lifestyle}

Irrespective of the aetiology of cirrhosis, alcohol and smoking cessation is advised. In addition, optimising sleep quality and psychological wellbeing (ie mindfulness and relaxation techniques) is essential in improving physiological reserve and muscle health.

\section{Nutrition}

The current clinical practice guidelines recommend caloric energy intake of 25-35 kcal/kg/day in compensated cirrhosis(Child-Pugh A) and 30-35 kcal/kg/day in decompensated cirrhosis (Child-Pugh $\mathrm{B}-\mathrm{C}) .^{12}$ In addition, protein intake of $1.2-1.5 \mathrm{~g} / \mathrm{kg} / \mathrm{day}$ in compensated cirrhosis, with this increasing up to $2.0 \mathrm{~g} / \mathrm{kg} /$ day in more severe cases of decompensation (eg in regular paracentesis for refractory ascites or hepatic encephalopathy) is recommended. BCAA have been studied with promising results and, while formal recommendations are pending, there appears to be strong rationale for their use in promoting muscle protein synthesis. ${ }^{3,10-12}$ A reduction in fasting times is imperative to combat accelerated
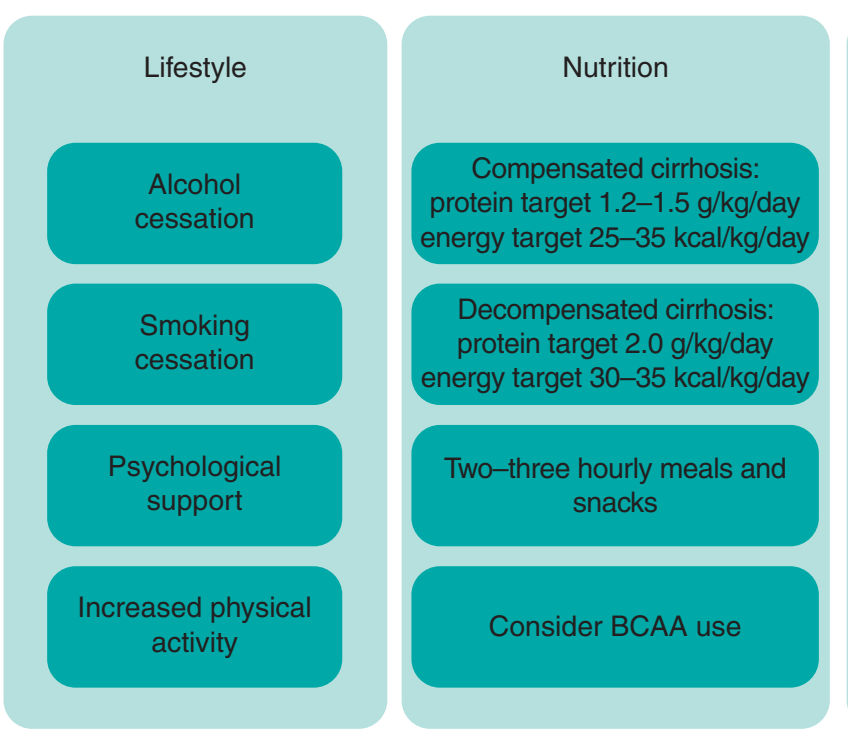

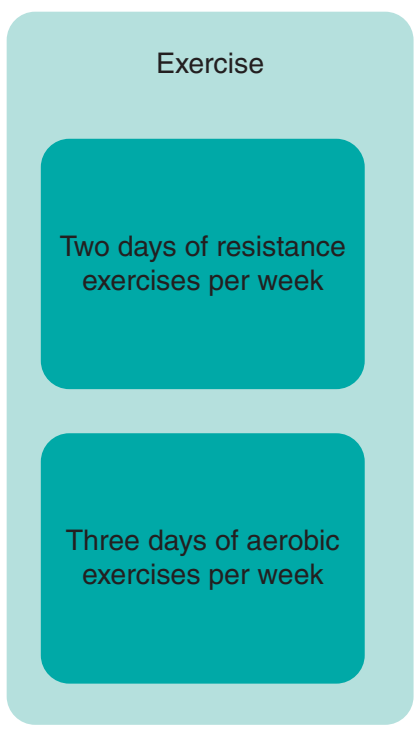

Fig 2. Overview of treatment of sarcopenia in liver cirrhosis. $\mathrm{BCAA}=$ branched-chain amino acids. 
starvation state, with regular pattern of 2-3 hourly meals and $50 \mathrm{~g}$ carbohydrate (+/- $20 \mathrm{~g}$ protein) snack prior to sleeping. ${ }^{1,5,10,11}$ It is also important to address any micronutrient deficiencies (such as vitamin $A, D, E$ and $K$ ) which can develop especially in cholestasis, and to address zinc deficiency in the presence of dysgeusia.

\section{Exercise}

Physical inactivity strongly predicts mortality in an array of chronic diseases. It is a key driver of sarcopenia in cirrhosis. Exercise has an impact on both cardiovascular fitness and increasing muscle mass and promoting muscle protein synthesis. Exercise has been shown to improve aerobic exercise capacity (6MWD), muscle function (SPPB), muscle mass (CT) and, importantly, quality of life in mainly compensated and, studied to lesser degree, decompensated cirrhosis. To date, no studies have studied survival benefit. In general, exercise programmes should consist of a combination of aerobic (3 days/week) and resistance exercises (2 days/week), performed to a moderate intensity (ie out of breath but able to talk) and ideally be remotely-monitored in the patient's own home.17,19,20

\section{Adjunct therapy}

Treatments to lower ammonia, hormonal treatment (testosterone) and micronutrient supplementation including vitamin $D$ have been areas of recent research. Myostatin antagonists have been proven to increased muscle mass in murine models, however translation into human studies is awaited. ${ }^{2,13}$ Furthermore, it is important to consider the effect of behaviour and need for psychological support to ensure adherence to management, avoidant behaviours and impact of low mood.

\section{Summary}

Sarcopenia affects many patients with cirrhosis, in particular those with liver failure. Early identification through routine imaging, easy-to-apply assessments and the observations with clinical suspicion is an integral part of the care of patients with cirrhosis. Management should be tailored to the patient's specific requirements and focus on high protein intake, regular mealtimes (including night-time snack), combination exercises, psychological support and optimisation of the underlying liver disease (ie ammonia-lowering therapy).

\section{Declaration}

The views expressed are those of the authors and not necessarily those of the NHS, the National Institute for Health Research nor the Department of Health and Social Care.

\section{References}

1 Carey EJ, Lai JC, Sonnenday C et al. A North American expert opinion statement on sarcopenia in liver transplantation. Hepatology 2019;70:1816-29.
2 Dasarathy S, Merli M. Sarcopenia from mechanism to diagnosis and treatment in liver disease. J Hepatol 2016;65:1232-44.

3 Ooi PH, Hager A, Mazurak VC et al. Sarcopenia in chronic liver disease: impact on outcomes. Liver Transpl 2019;25:1422-38.

4 Montano-Loza AJ, Duarte-Rojo A, Meza-Junco J et al. Inclusion of sarcopenia within MELD (MELD-Sarcopenia) and the prediction of mortality in patients with cirrhosis. Clin Transl Gastroenterol 2015;6:e102.

5 Tandon P, Low G, Mourtzakis M et al. A model to identify sarcopenia in patients with cirrhosis. Clin Gastroenterol Hepatol 2016;14:1473-80.e3.

6 Tandon P, Ney M, Irwin I et al. Severe muscle depletion in patients on the liver transplant wait list: its prevalence and independent prognostic value. Liver Transp/ 2012;18:1209-16.

7 Williams R, Alexander G, Aspinall R et al. Gathering momentum for the way ahead: fifth report of the Lancet Standing Commission on Liver Disease in the UK. Lancet 2018;392:2398-412.

8 Welch N, Dasarathy J, Runkana A et al. Continued muscle loss increases mortality in cirrhosis: Impact of aetiology of liver disease. Liver International 2020;40:1178-88.

9 Koo BK, Kim D, Joo SK et al. Sarcopenia is an independent risk factor for non-alcoholic steatohepatitis and significant fibrosis. J Hepatol 2017;66:123-31.

10 Plauth M, Bernal W, Dasarathy S et al. ESPEN guideline on clinical nutrition in liver disease. Clin Nutr 2019;38:485-521.

11 European Association for the Study of the Liver. EASL Clinical Practice Guidelines on nutrition in chronic liver disease. J Hepatol 2019;70:172-93.

12 Dhaliwal A, Towey J, Lord JM, Armstrong M, Elsharkawy AM. Nutrition in liver cirrhosis: a case-based overview. Frontline Gastroenterology 2020;11:155-61.

13 Ebadi M, Bhanji RA, Mazurak VC, Montano-Loza AJ. Sarcopenia in cirrhosis: from pathogenesis to interventions. J Gastroenterol 2019:54:845-59.

14 Dasarathy S. Cause and management of muscle wasting in chronic liver disease. Curr Opin Gastroenterol 2016;32:159-65.

15 Bhanji RA, Narayanan P, Allen AM, Malhi H, Watt KD. Sarcopenia in hiding: The risk and consequence of underestimating muscle dysfunction in nonalcoholic steatohepatitis. Hepatology 2017;66: 2055-65.

16 El Sherif O, Dhaliwal A, Newsome PN, Armstrong MJ. Sarcopenia in nonalcoholic fatty liver disease: new challenges for clinical practice. Expert Rev Gastroenterol Hepatol 2020;14:197-205.

17 Lai JC, Sonnenday CJ, Tapper EB et al. Frailty in liver transplantation: An expert opinion statement from the American Society of Transplantation Liver and Intestinal Community of Practice. Am J Transplant 2019;19:1896-906.

18 Van Jacobs AC. Frailty assessment in patients with liver cirrhosis. Clinical Liver Disease 2019;14:121-5.

19 Williams FR, Berzigotti A, Lord JM, Lai JC, Armstrong MJ. Review article: impact of exercise on physical frailty in patients with chronic liver disease. Aliment Pharmacol Ther 2019;50:988-1000.

20 Tandon P, Ismond KP, Riess K et al. Exercise in cirrhosis: Translating evidence and experience to practice. J Hepatol 2018;69:1164-77.

Address for correspondence: Dr Amritpal Dhaliwal, Liver Unit, Queen Elizabeth Hospital Birmingham, Mindelsohn Way, Edgbaston, Birmingham B15 2GW, UK.

Email: a-dhaliwal@live.co.uk 